\title{
CASO RADIOLOGICO PARA DIAGNOSTICO
}

Drs. Francisca Fuentes $D^{(1)}$, Camila Urzúa $F^{(1)}$, Marcelo Castro $S^{(2)}$, Int. Verónica López ${ }^{(3)}$.

1. Servicio de Diagnóstico por Imágenes, Hospital Militar de Santiago - Chile.

2. Jefe Servicio de Diagnóstico por Imágenes, Hospital Militar de Santiago - Chile.

3. Interna de Medicina, Universidad de los Andes, Chile.

\section{Caso clínico}

Hombre de 67 años con antecedente de leucemia linfática crónica diagnosticada hace un año, en quimioterapia, púrpura trombocitopénico idiopático (esplenectomizado), y diabetes esteroidal. Consulta en el servicio de urgencia por cuadro de 3 meses de evolución de disnea leve a moderada, que en los últimos 3 días llega a ser de mínimos esfuerzos, asociándose a tos seca y fiebre de hasta $38,5^{\circ} \mathrm{C}$. Al examen físico destaca: taquicardia, polipnea, palidez de mucosas y crépitos basales izquierdos (afebril y normotenso). Los exámenes de laboratorio iniciales muestran anemia microcítica hipocrómica (hematocrito: $26 \%$ ), leucocitosis de 20.600 con $78 \%$ de neutrófilos, y VHS $51 \mathrm{~mm} / \mathrm{hr}$. Las pruebas de función renal y de coagulación se encontraban normales. La radiografía de tórax evidencia múltiples focos de condensación bilaterales. Se ingresa con diagnóstico de neumonía multifocal y se inicia tratamiento antibiótico intravenoso. La TC de tórax muestra fenómenos parenquimatosos pulmonares bilaterales, que se interpretan como de origen infeccioso, y signos de fibrosis pulmonar bilateral, con un patrón inespecífico (probablemente secundaria a drogas citotóxicas). La TC de abdomen y pelvis evidencia los hallazgos que se observan en la Figura 1.

¿Cuál es su diagnóstico?
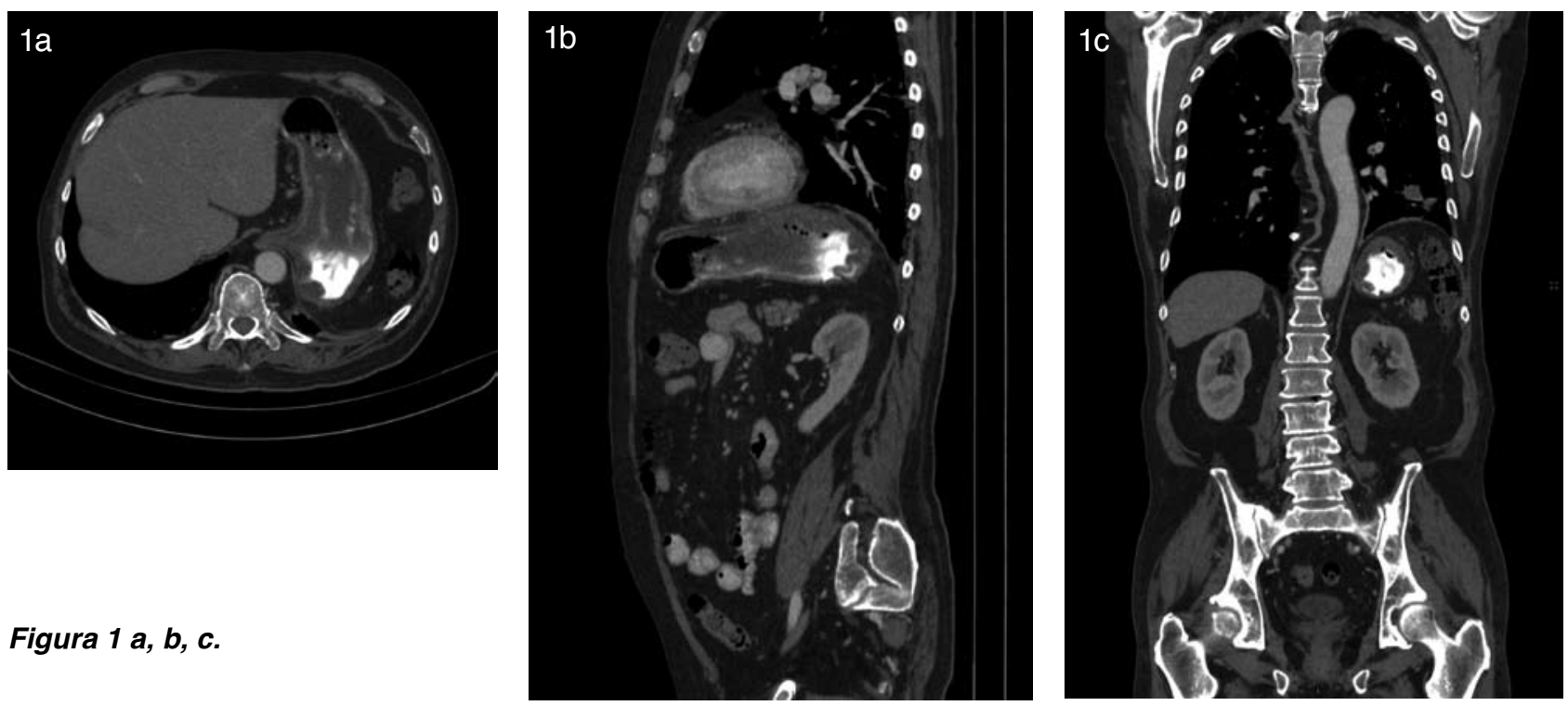

Figura 1 a, $b, c$. 


\section{RESULTADO CASO RADIOLOGICO PARA DIAGNOSTICO}

\section{Drs. Francisca Fuentes $D^{(1)}$, Camila Urzúa $F^{(1)}$, Marcelo Castro $S^{(2)}$, Int. Verónica López ${ }^{(3)}$.}

1. Servicio de Diagnóstico por Imágenes, Hospital Militar de Santiago - Chile.

2. Jefe Servicio de Diagnóstico por Imágenes, Hospital Militar de Santiago - Chile.

3. Interna de Medicina, Universidad de los Andes, Chile.

Viene de la página 100.
Diagnóstico:

Mucormicosis gástrica
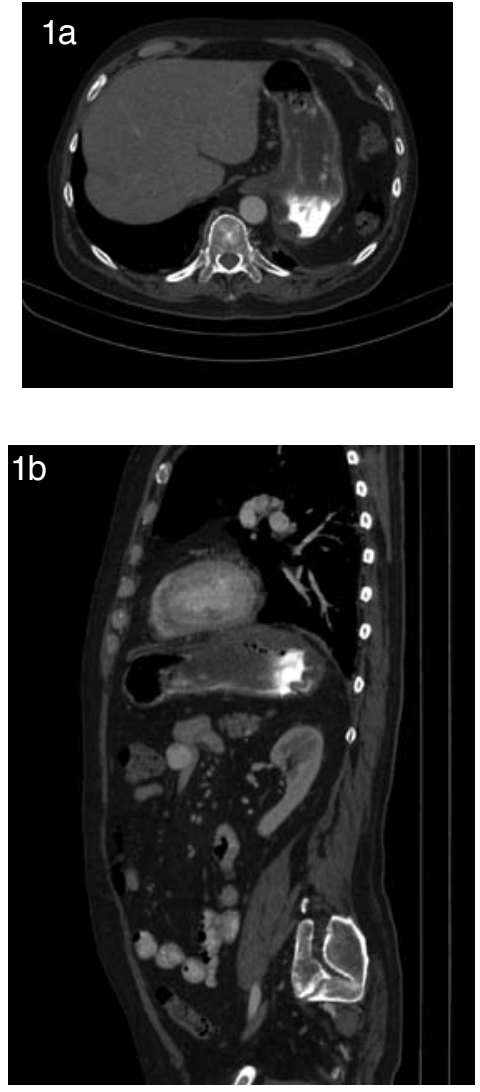

En la TC de abdomen y pelvis destaca engrosamiento parietal del fondo gástrico, asociado a linfonodos aumentados de tamaño en el ligamento gastrohepático (Figura 1). La endoscopía digestiva alta evidencia lesión corporal ulcerada de aspecto neoplásico (Bormann II), que se biopsia (Figura 2). El paciente evoluciona favorablemente, por lo que es dado de alta con tratamiento antibiótico oral, para luego decidir conducta de la lesión gástrica. El reporte histopatológico (Figuras 3,4 ) mostró gastritis crónica reagudizada con colonias fúngicas, que más tarde, el cultivo confirmaría como hongo filamentoso Rhyzopus Cryzae, compatibles con Mucormicosis Gástrica. Reingresa para inicio de Anfotericina B sistémica. Inicialroente evoluciona con extensión del compromiso gástrico y luego desarrolla una embolía pulmonar (confirmada con angioTC), lo que obliga a iniciar terapia anticoagulante, a pesar del alto riesgo. Dos días después, presenta hemorragia digestiva alta con compromiso hemodinámico que responde a manejo médico. Finalmente, desarrolla insuficiencia respiratoria progresiva y falla renal aguda, por lo que se suspende la terapia antifúngica. El paciente fallece un mes después del diagnóstico.

Figura 1. a) Corte axial y reconstrucciones de TC de tórax, abdomen y pelvis: b) sagital y c) coronal, con contraste oral y endovenoso. Destaca engrosamiento parietal del fondo gástrico, en estómago parcialmente distendido.

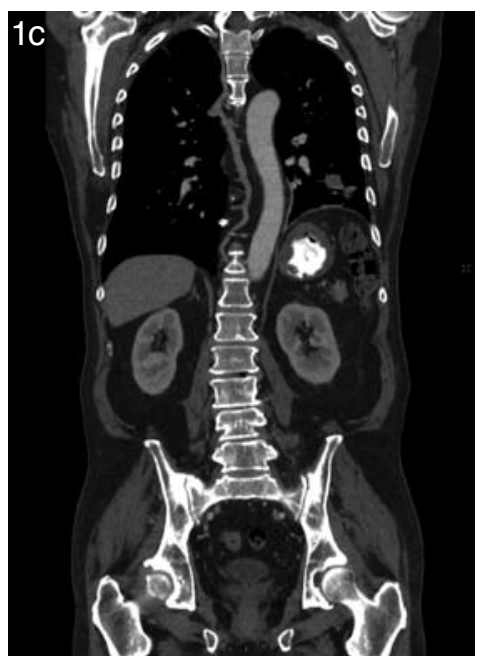




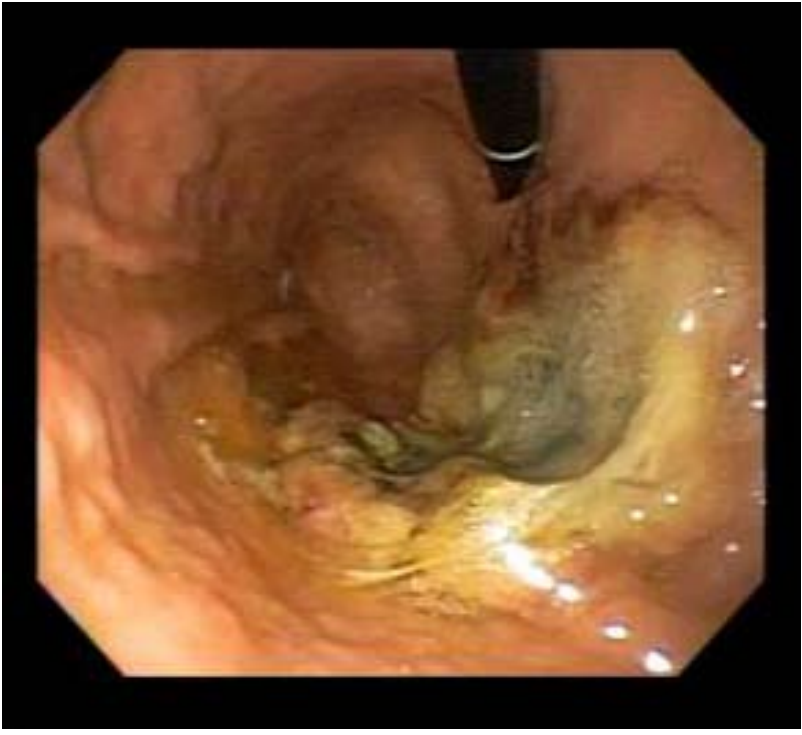

Figura 2. Endoscopía digestiva alta. Lesión ulcerada tipo Bormann II.

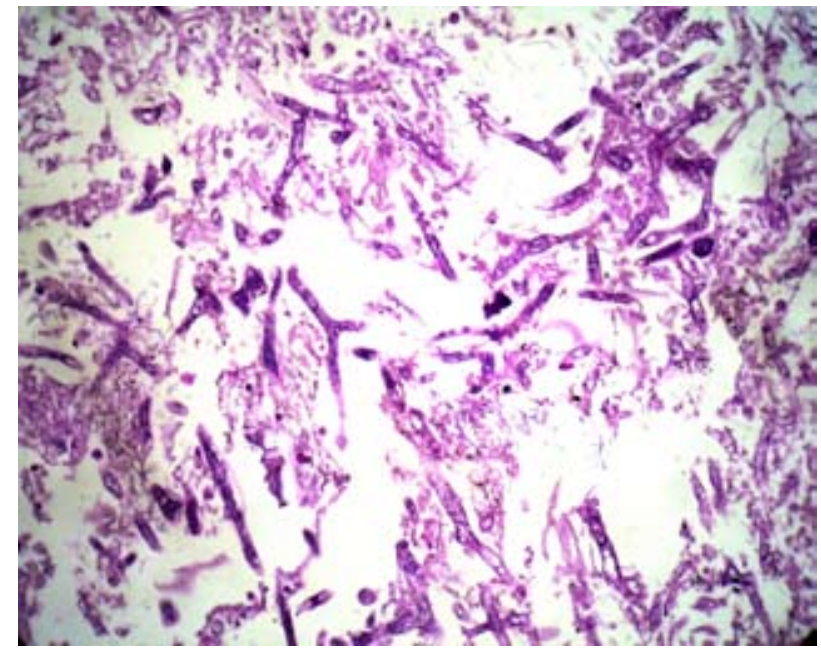

Figura 4. Mediante tinción PAS (+), las múltiples hifas son más evidentes.

\section{Discusión}

La evaluación del estómago en TC está limitada por varios factores como: peristalsis, distensión insuficiente, inadecuado volumen de contraste oral y retención de alimentos. El uso de agentes efervescentes, antiespasmódicos y adecuada cantidad de contraste oral (neutro o positivo) favorecen su evaluación ${ }^{(1)}$. Se considera que el grosor normal de la pared del estómago debidamente distendido en TC es 7-10 mm. Muchas veces, debido a la falta de distensión, se puede pasar por alto la presencia de anomalías parietales o interpretar erróneamente pseudotumores como neoplásicos, hallazgo muy frecuente de observar en la unión gastroesofágica. Se han descrito múltiples causas de engrosamiento

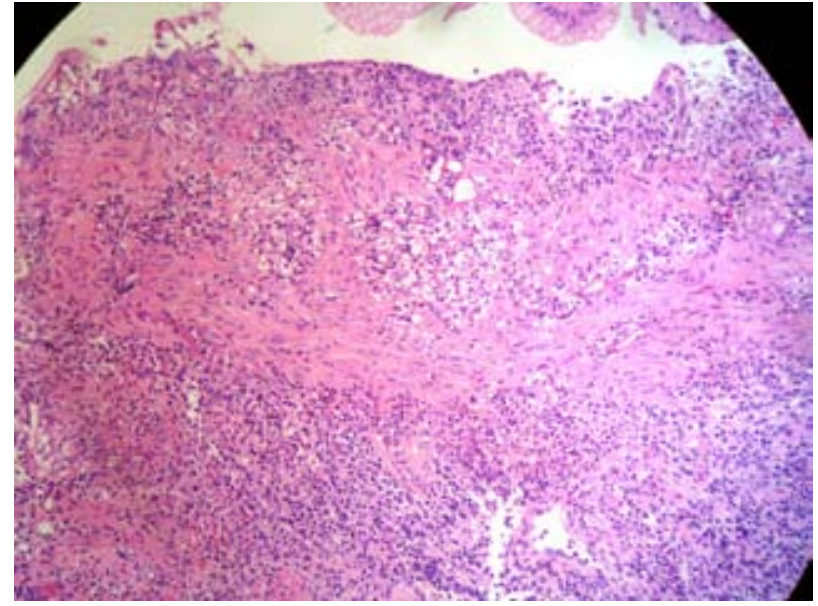

Figura 3. Biopsia gástrica. Muestra una lesión ulcerada de la mucosa en cuyo espesor se evidencian numerosas hifas, correspondientes a mucormicosis (HE 40x).

parietal gástrico, entre las cuales se encuentran:

- Neoplásicas (adenocarcinoma, linfoma, metástasis o sarcoma de Kaposi),

- Inflamatorias (gastritis por Helicobacter Pylori)

- Gastroenteritis eosinofílica

- Infecciosas (TBC y sífilis)

- Enfermedad de Crohn y sarcoidosis (raras)(2).

El engrosamiento parietal es la alteración más comúnmente detectada mediante TC en pacientes con sospecha de patología gástrica. Se considera patológico un grosor parietal mayor a 1 centímetro. Sin embargo, este hallazgo es altamente sensible pero poco específico $(50 \%)$ en la detección de lesiones malignas o potencialmente malignas, por lo que para evaluar adecuadamente una lesión deben tomarse en cuenta otros parámetros como: simetría, distribución y refuerzo con el medio de contraste intravascular. El análisis de estas características en conjunto aumenta la sensibilidad y especificidad diagnósticas por lo que la pesquisa de un engrosamiento parietal focal excéntrico que refuerza con contraste tiene elevada sensibilidad (92\%) y especificidad en la detección de lesiones malignas ${ }^{(1)}$. Debido a que sólo un $36 \%$ de los pacientes con neoplasias gástricas tienen engrosamiento parietal, la TC no debe utilizarse para la detección de neoplasias gástricas y, las alteraciones que se pesquisan a la TC deben ser confirmadas con endoscopía.

\section{Patogenia}

La mucormicosis es una rara infección fúngica oportunista descrita por primera vez por Paultauf en 1885. Es la segunda micosis más común causada por hongos filamentosos, después de la aspergilosis. El género de orden Mucorales constituye la tercera causa de infecciones fúngicas invasivas, luego del 
Aspergilus y Candida sp. Son hongos filamentosos ubicuos de baja patogenicidad intrínseca que pueden producir infecciones graves en pacientes inmunocomprometidos. Más frecuentemente es producida por la especie Rizopus (47\%), seguida por Mucor, Cunninghamella, Apphysomyces, Absidia, saksenaea y Rhizomucor ${ }^{(3)}$. Constan de una típica forma infectante llamada esporangioespora que sería ingerida directamente por vía oral y una hifa angioinvasiva, que es la responsable de la diseminación e invasión de tejidos. A la histopatología, la mucormicosis gastrointestinal (GI) presenta característicamente angioinvasión por penetración directa de la pared y crecimiento a través de ésta, seguida de trombosis y necrosis secundaria de los tejidos. Estos hongos tienen además capacidad de extraer fierro del huésped mediante quelantes del fierro y permeasas de alta afinidad, como la ferriziferrina, que es esencial en los procesos de replicación y sobrevida del hongo.

\section{Epidemiología}

Aunque clásicamente se ha descrito a la Candida $\mathrm{sp}$, como la causa más frecuente de infecciones fúngicas invasivas, en los últimos años se ha observado un aumento de la incidencia de infecciones causadas por Aspergillus y hongos filamentosos, atribuyéndose a la mayor sobrevida de inmunosuprimidos y al uso de azoles (especialmente Voriconazol) por largos períodos en la profilaxis de Candida $\mathrm{sp}^{(6)}$, entre otros. Las infecciones fúngicas invasivas provocan un incremento en la morbimortalidad de los pacientes inmunodeprimidos.

\section{Clínica}

El espectro clínico de las micosis por Mucorales va desde formas cutáneas localizadas, hasta enfermedad diseminada. En general, la severidad de la infección depende de la capacidad de invasión vascular, que causa necrosis de los tejidos. La prevalencia de las distintas formas de compromiso es variable, dependiendo de las condiciones clínicas de base. Las formas más comunes son rinocerebral, maxilofacial y pulmonar, seguidas por la gastrointestinal, con una prevalencia de $7 \%$. Aunque todo el tubo digestivo es susceptible de infectarse, principalmente se compromete estómago, colon, intestino delgado y esófago, en orden descendente de frecuencia. Las manifestaciones clínicas iniciales de mucormicosis gástrica son: dolor, distensión abdominal, fiebre y hemorragia. Adicionalmente pueden observarse linfoadenopatías regionales, lo que no constituye un hallazgo específico. Al ingreso, nuestro paciente presentaba disnea y fiebre, que podrían explicarse con el cuadro respiratorio.

Se han identificado tres etapas de compromiso gastrointestinal: colonización, que puede estar en relación a lesiones preexistentes como úlceras, infiltración y finalmente invasión vascular. Morfológicamente se presenta en una etapa inicial como lesión colonizada sin invasión del tejido circundante; luego se produce la invasión y la lesión se extiende, provocando un marcado aumento de volumen e induración periférica, con una superficie central vellosa o aterciopelada, descolorida o con grandes placas verdosas y necróticas que simulan una neoplasia.

\section{Factores asociados}

Se han descrito como factores predisponentes: sepsis, quemaduras extensas, malnutrición, edades extremas, pica, ulcera gástrica ${ }^{(4)}$, terapia corticoidal crónica, uso prolongado de antibióticos, enfermedad sistémica severa, inmunosupresión y enfermedades como: diabetes mellitus, cirrosis, neoplasias hematológicas, trasplantes e insuficiencia renal. La mucormicosis frecuentemente se origina en el contexto de neoplasias hematológicas, principalmente leucemia aguda $(78 \%)$ y linfoma ${ }^{(5)}$, debido probablemente a neutropenia severa y prolongada quimioterapia mieloablativa; tratamiento se puede observar en relación con tratamiento inmunosupresor en pacientes trasplantados. Los enfermos portadores de neutropenia tienen mayor riesgo de desarrollar infecciones diseminadas; un $40 \%$ de los pacientes hematológicos con mucormicosis desarrollará diseminación ${ }^{(5)}$.

\section{Diagnóstico y pronóstico}

La mucormicosis tiene una elevada tasa de mortalidad (>98\%); sólo un $25 \%$ de los pacientes con compromiso $\mathrm{Gl}$ son diagnosticados pre-mortem ${ }^{(3)}$. El diagnóstico se hace mediante biopsia del tejido infectado, que evidencia la presencia del hongo. La citología no está recomendada ya que tiene bajo rendimiento en aislar el microorganismo. Técnicas de laboratorio como la serología y PCR actualmente contribuyen al diagnóstico.

\section{Tratamiento}

Consta de tres pilares fundamentales: terapia antifúngica, extirpación quirúrgica del tejido infectado y manejo médico de la neutropenia. Para un manejo exitoso, es necesario además iniciar en forma oportuna el tratamiento y otorgar un apoyo metabólico agresivo. La anfotericina B de uso sistémico es la terapia antifúngica de elección, sin embargo su uso está limitado debido a efectos adversos, como el deterioro grave de la función renal que frecuentemente determina la suspensión del tratamiento, como sucedió en nuestro caso. La anfotericina B liposomal, es más eficaz ya que permite administrar mayores dosis con menos efectos adversos. Nuevas terapias propuestas son Posaconazol, en caso de resistencia a anfotericina, y deferiprone que inhibe la captación de fierro por parte de los Rizopus. También se ha demostrado utilidad la terapia coadyuvante con oxígeno hiperbárico. 


\section{Conclusiones}

La mucormicosis gástrica es una infección fúngica grave, que debe sospecharse en un paciente inmunodeprimido, frente al hallazgo de aumento del grosor parietal gástrico. Si bien su pronóstico es bastante ominoso, es necesario sospecharla para lograr un diagnóstico oportuno y así aumentar la probabilidad de éxito terapéutico.

La TC presenta limitaciones en el estudio del estómago, que es importante conocer. El desafío está en saber interpretar las imágenes y diferenciar un estómago normal de uno patológico, que requerirá mayor análisis y estudios complementarios.

\section{Bibliografías}

1. Insko E, Levine M, Birnbaum B, Jacobs J. Bening and Malignant Lesions of the Stomach: Evaluation of CT Criteria for Differentiation. Radiology 2003; 228:166-171.

2. Brant W. Stomach and Duodenum. Brant W, Helms H. Fundamentals of Diagnostic Radiology. Tercera edición. Lippincott Williams and Wilkins; 2007; 816-831.

3. Shiva Prasad BN, Shenoy A, Nataraj KS. Primary Gastrointestinal Mucormycosis in an Immunocompetent Person. J Postgrad Med 2008; 45: 211- 213.

4. Benjamin SPE. Primary Gastric Mucormycosis: Role of Preexisting Ulcerative and Erosive Lesions. J Postgrad
Med 2009; 55: 73-74.

5. Pagano L, Offidani M, Fianchi L, Nosari A, Candoni A, Piccardi M et al. Mucormycosis in Hematologic Patients. Haematologica 2004; 89: 207-214.

6. Castón-Osorio JJ, Rivero A, Torre-Cisneros J. Epidemiology of Invasive Fungal Infection. Int J Antimicrob Agents 2008; 103-109.

7. Desai R, Tagliabue J, Wegryn S, Einstein D. CT EvaIuation of Wall Thickening in the Alimentary Tract. Radiographics 1991; 11: 771-783.

8. Einollahi B, Lessan-Pezeshki M, Pourfarziani V, Nemati E, Nafar M, Pour-Reza-Gholi F et al. Invasive Fungal Infections Following Renal Transplantation: A Review of 2410 recipients. Ann Transplant 2008; 13(4): 55-58.

9. Jung L, Choi H, Yoo J, Kang S, Lee K. Emphysematous Gastritis Associated with Invasive Gastric Mucormycosis: A Case Report. J Korean Med Sci 2007; 22: 923-927.

10. Song K, Kang W, Park Ch, Choi Y, Rha S, Park Ch. Mucormycosis Resulting in Gastric Perforation in a Patient with Acute Myelogenous Leukemia: Report of a Case. Surg Today 2006; 36: 831-834.

Fuentes Fy cols. Mucormicosis gástrica. Rev Chil Radiol 2010; 16(3): 100, 159-162.

Correspondencia: Dra. Francisca Fuentes del Pozo franfuentesd@gmail.com

Trabajo recibido el 06 de noviembre de 2009, aceptado para publicación el 09 de agosto de 2010. 